\title{
Nonsymmetrical Separations in Thermal Diffusion Columns
}

\author{
M. F. L. S. MORGADO, J. de D. R. S. PINHEIRO, and
} J. J. B. ROMERO

CENTRO DE QUIMICA PURA E APLICADA

UNIVERSIDADE DO MINHO

4719 BRAGA, PORTUGAL

\section{T. R. BOTT}

CHEMICAL ENGINEERING DEPARTMENT

THE UNIVERSITY OF BIRMINGHAM

BIRMINGHAM B15 2TT, UNITED KINGDOM

\begin{abstract}
The influence of the feed composition upon the actual degrees of separation attained at the top and bottom sections of a thermogravitational column is discussed using the classical phenomenological theory of Furry, Jones, and Onsager. It is shown that, except for a feed composition of $C_{0}=0.5$ (mass fraction), the separation profile is nonsymmetric, i.e., the separations in the top and bottom sections of the column are nonsymmetric with respect to the feed composition, the asymmetry increasing as the feed composition moves away from $C_{0}=0.5$. An equation for the determination of the optimum feed location as a function of the feed composition is derived.
\end{abstract}

\section{INTRODUCTION}

The degree of separation attained by a thermal diffusion column is usually expressed as

$$
\Delta=\left|C_{T}-C_{B}\right|
$$

where $\Delta$ is the degree of separation and $C_{T}$ and $C_{B}$ represent the mass fraction concentration of the reference component at the top and bottom ends of the column, respectively.

In some instances it is convenient to consider separately the upper and

897

Copyright $\odot 1981$ by Marcel Dekker, Inc. 
lower sections of the column (also referred to as enriching and stripping sections) (1) in which case use is made of the so-called upper or top degree of separation, $\Delta_{T}$, and lower or bottom degree of separation, $\Delta_{B}$, defined as

$$
\begin{aligned}
& \Delta_{T}=\left|C_{T}-C_{0}\right| \\
& \Delta_{B}=\left|C_{B}-C_{0}\right|
\end{aligned}
$$

and, obviously,

$$
\Delta=\Delta_{T}+\Delta_{B}
$$

It is generally accepted that, for an ideal column, the values of $\Delta_{T}$ and $\Delta_{B}$ should be identical or, in other words, that the separation should be symmetrical with regard to the initial concentration, $C_{0}(2-4)$. As a consequence, the column design with a feed-line halfway along its length becomes well established and the reported asymmetrical separations experimentally observed are most often considered as deviations from the ideal behavior due, mainly, to some unequal heating along the column or annular eccentricities $(5,6)$.

It is therefore of importance to determine to what extent is the asymmetry in separation a deviation from ideality or a consequence of the thermogravitational process. For this purpose, use is made of the phenomenological theory of Furry, Jones, and Onsager (7) as presented by Romero (8).

\section{BASIC EQUATIONS}

According to the theory $(7,8)$, the transport of the reference component along the column (Fig. 1) is governed by the equation

$$
W=H C(1-C)-\left(K_{\varepsilon}+K_{d}\right) \frac{\partial C}{\partial Z}
$$

where

$$
\begin{gathered}
H=\frac{\alpha \beta \rho g B(\Delta T)^{2}(2 a)^{3}}{6 ! T_{a v} \mu} \\
K_{C}=\frac{\beta^{2} \rho g^{2} B(\Delta T)^{2}(2 a)^{7}}{9 ! D \mu^{2}} \\
K_{d}=(2 a) \rho D B
\end{gathered}
$$

and all other symbols are defined in the "Symbol" section. 


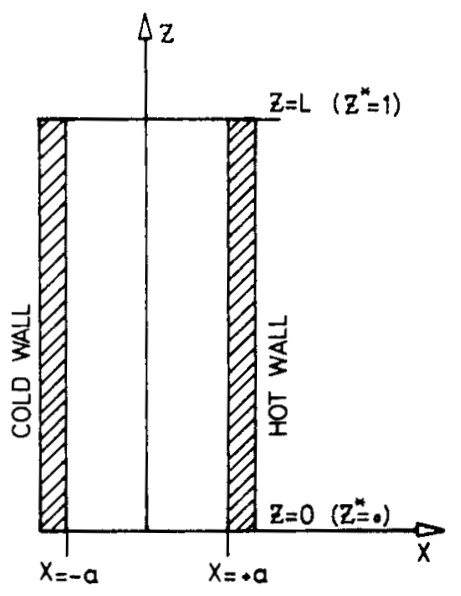

FIG. 1. Thermogravitational column.

By definition, $W=0$ at the steady state and Eq. (5) becomes

$$
0=H C_{\infty}\left(1-C_{\infty}\right)-\left(K_{c}+K_{d}\right) \frac{\partial C_{\infty}}{\partial Z}
$$

where the subscript $\infty$ identifies "steady-state values."

Integration of Eq. (9) subjected to the condition

$$
C_{\curvearrowright}=C_{B \infty} \quad \text { at } \quad L=0
$$

yields

$$
C_{\infty}=\frac{e^{\lambda Z^{*}} C_{B^{\infty}}}{e^{\lambda Z^{*}} C_{B \infty}-C_{B \infty}+1}
$$

where

$$
Z^{*}=Z / L
$$

and

$$
\lambda=\frac{H L}{K_{c}+K_{d}}
$$

or, since in most situations $K_{c} \gg K_{d}, \lambda$, the separation potential, is given by 


$$
\lambda=\frac{H L}{K_{c}}=504 \frac{\alpha D \mu L}{\beta g T_{a v^{\prime}}(2 a)^{4}}
$$

It is possible to relate $C_{\infty}$ to the initial concentration $C_{0}$ through a mass balance for the reference component

$$
\int_{0}^{1} C_{\infty} d Z^{*}=C_{0}
$$

Introducing the value of $C_{\infty}$ from Eq. (11) into Eq. (14) and integrating, one obtains after rearranging

$$
C_{\infty}=\frac{e^{\lambda Z^{*}}\left(e^{\lambda C_{0}}-1\right)}{e^{\lambda Z^{*}}\left(e^{\lambda C_{0}}-1\right)+\left(e^{\lambda}-e^{\lambda C_{0}}\right)}
$$

The concentration at the top and bottom extremities of the column are simply obtained from Eq. (15), making $Z^{*}=1$ and $Z^{*}=0$, yielding respectively:

$$
C_{T_{\infty}}=\frac{e^{-\lambda C_{0}}-1}{e^{-\lambda}-1}, \quad C_{B \times}=\frac{e^{\lambda C_{0}}-1}{e^{\lambda}-1}
$$

Combining Eqs. (15) and (16), one may obtain the expression for the steady-state degree of separation, $\Delta_{\infty}$ :

$$
\Delta_{\infty}=\left|C_{T \infty}-C_{B \infty}\right|=\frac{\left(e^{\lambda C_{0}}-1\right)\left(e^{\lambda}-e^{\lambda C_{0}}\right)}{e^{\lambda C_{0}}\left(e^{\lambda}-1\right)}
$$

Equation (17) is exactly the same as obtained previously by several authors $(7,10)$, confirming, therefore, the validity of the expressions obtained for the dependence of $C_{\star}$ on $Z^{*}$.

\section{SEPARATION ASYMMETRY}

The symmetry condition

$$
\Delta_{T}=\Delta_{B}
$$

or

$$
C_{T}-C_{0}=C_{0}-C_{B}
$$


may be written, in accordance with Eq. (16), as

$e^{\lambda\left(1-C_{0}\right)}+e^{-\lambda\left(1-C_{0}\right)}-e^{\lambda}-e^{-\lambda}-e^{-\lambda C_{0}}-e^{\lambda C_{0}}+2 C_{0}\left(e^{\lambda}+e^{-\lambda}\right)=4\left(C_{0}-2\right)$

Analysis of Eq. (18) shows that the condition will hold only in two singular circumstances:

(1) $\lambda=0$, in which case $C_{T^{\infty}}=C_{B^{\infty}}=C_{0}$, i.e., there is no separation

(2) $C_{0}=1 / 2$ (for any $\lambda$ )

One may therefore conclude that the transport equation derived by Furry, Jones, and Onsager (7) does, indeed, predict that all separations are asymmetrical, except for the case of $C_{0}=0.5$.

A measure of the actual asymmetry may be given by an asymmetry-factor, $A_{f}$, defined as

$$
A_{f}=\ln \frac{\Delta T}{\Delta B}=\ln \frac{C_{T}-C_{0}}{C_{0}-C_{B}}
$$

It may be seen that, theoretically, $A_{f}=0$ for $C_{0}=0.5$. For $C_{0} \neq 0.5$, the value of $A_{f}$ depends both on $C_{0}$ and $\lambda$, since $C_{B \infty}$ and $C_{T^{\infty}}$ are also functions of $C_{0}$ and $\lambda$.

The dependence of $C_{B \infty}, C_{T^{\infty}}$, and $A_{f}$ on $\lambda$ is illustrated in Fig. 2 for $C_{0}=$ 0.7 and $C_{0}=0.9$. As one would expect, $A_{f}$ increases as $\lambda$ increases and as $C_{0}$ moves away from the mid-value of 0.5 .

It is of importance to determine the exact section along the column length in which the actual average concentration is equal to the initial concentration. This section defines the optimum feed point which minimizes the time required to attain the steady-state (or that maximizes separation in continuous operation) since in this case no disturbance is introduced in the concentration profile along the column length.

From Eq. (15) one may get

$$
Z^{*}=\frac{1}{\lambda} \ln \left(\frac{C_{\infty}}{1-C_{\infty}} \frac{e^{\lambda}-e^{\lambda C_{0}}}{e^{\lambda C_{0}}-1}\right)
$$

Thus the value of $Z^{*}$ at which $C_{\infty}=C_{0}$ is

$$
\left.Z^{*}\right|_{C=C_{0}}=\frac{1}{\lambda} \ln \left(\frac{C_{0}}{1-C_{0}} \frac{e^{\lambda}-e^{\lambda C_{0}}}{e^{\lambda C_{0}}-1}\right)
$$




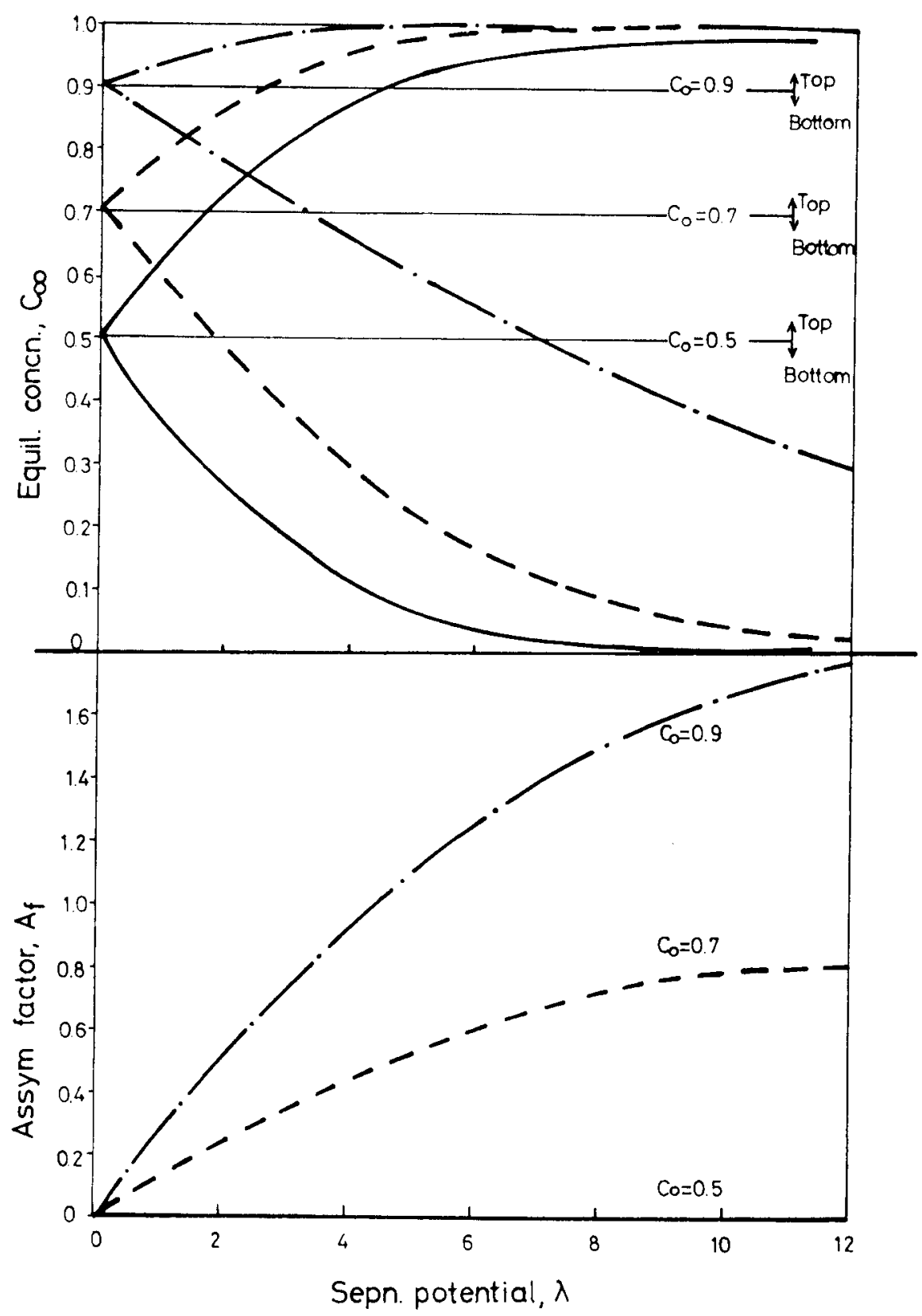

FIG. 2. Equilibrium concentrations at the top and bottom extremities of the column versus separation potential for different feed compositions. 
On the other hand, at half-length of the column, the actual average concentration at the steady-state is simply obtained from Eq. (15) by making $Z^{*}=1 / 2$;

$$
\left.C_{\infty}\right|_{Z^{*}=1 / 2}=\frac{e^{\lambda / 2}\left(e^{\lambda C_{0}}-1\right)}{e^{\lambda / 2}\left(e^{\lambda C_{0}}-1\right)+\left(e^{\lambda}-e^{\lambda C_{0}}\right)}
$$

Equation (22) provides a mean of testing the actual deviation from ideality in most columns, since it is free from any end effects that may eventually affect $C_{T}$ or $C_{B}$.

Examples of the effect of $\lambda$ on the actual values of $\left.Z^{*}\right|_{C=C_{0}}$ and $\left.C_{\infty}\right|_{Z^{*}=1 / 2}$ are shown in Figs. 3 and 4 for an initial concentration of $C_{0}=0.9$ (or $C_{0}=$ 0.1 ), $C_{0}=0.7$ (or $C_{0}=0.3$ ), and $C_{0}=0.5$.

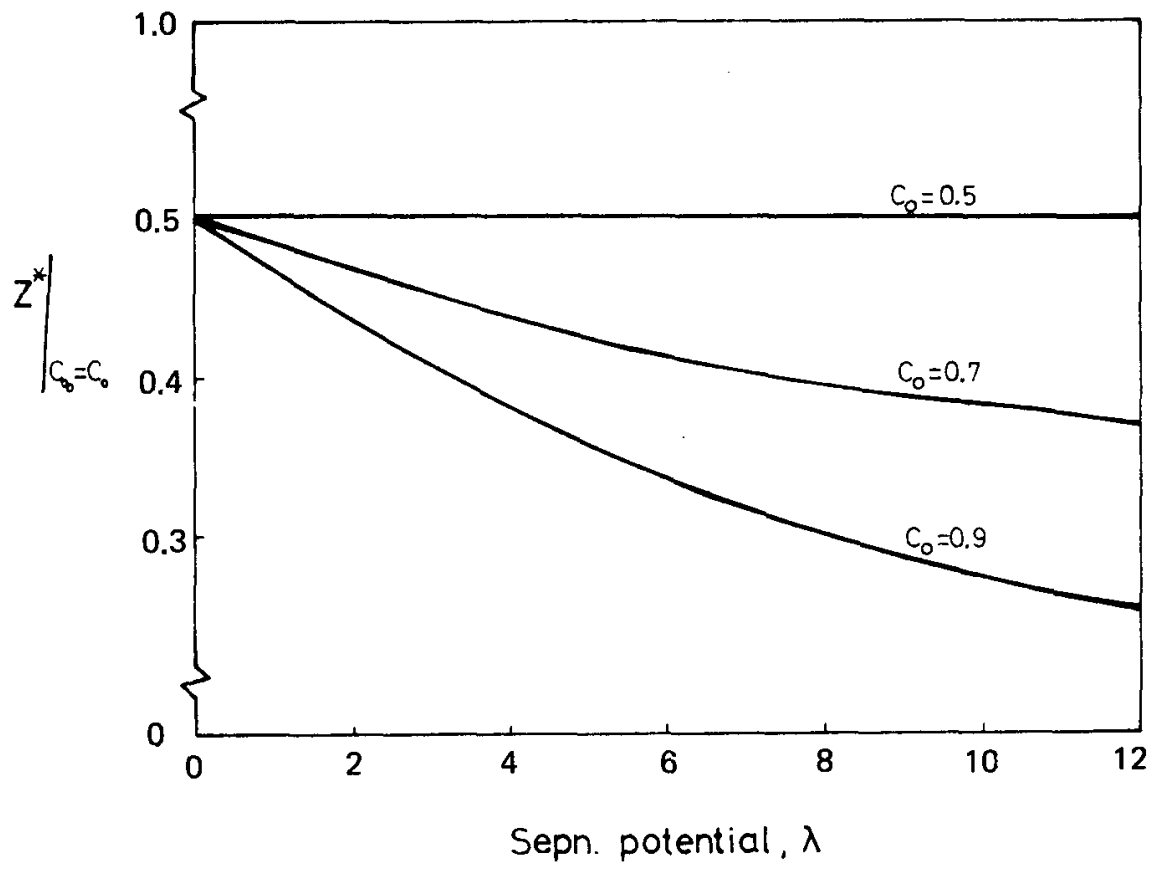

F1G. 3. Location of the column section for which the equilibrium composition coincides with the feed composition as a function of the separation potential for different feed compositions. 


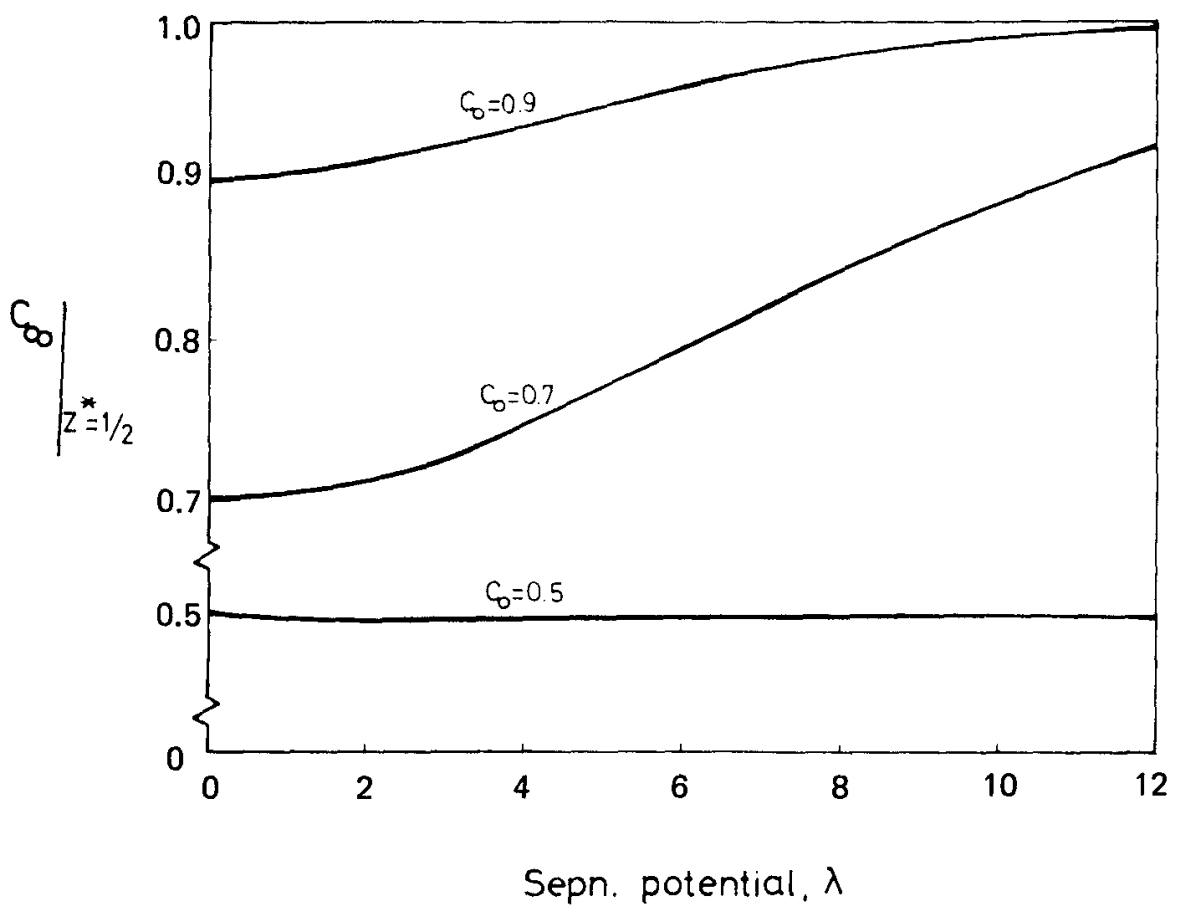

FIG. 4. Equilibrium composition at the midsection of the column versus separation potential for different feed compositions.

\section{CONCLUSIONS}

The asymmetry of separation, i.e., $C_{T}-C_{0} \neq C_{0}-C_{B}$, does not necessarily imply a deviation from ideality. On the contrary, from the phenomenological theory of Furry, Jones, and Onsager, it is shown that asymmetric separations will occur if and when $C_{0} \neq 0.5$.

The column section at which the average concentration is equal to the initial concentration does not coincide with the half-length of the column, except for $C_{0}=0.5$. That section which corresponds to the optimum feed location is a function of both the initial concentration and the separation potential as shown by Eq. (21).

\section{SYMBOLS}

a one-half of the annulus width

$A_{f} \quad$ asymmetry factor defined by Eq. (19) 
$B \quad$ column width in the $Y$-direction

$C$ mass fraction concentration of the reference component

$C_{0} \quad$ initial composition

$D$ ordinary diffusion coefficient

$g$ gravitational acceleration

$H$ transport coefficient defined by Eq. (6)

$K_{c} \quad$ transport coefficient defined by Eq. (7)

$K_{d} \quad$ transport coefficient defined by Eq. (8)

$L \quad$ column length

$T_{\text {av }} \quad$ average absolute temperature

$\Delta T \quad$ temperature difference between the hot and cold walls of the column

$W \quad$ mass flow rate of the reference component in the $Z$-direction

$Z^{*} \quad$ dimensionless length, $Z^{*}=Z / L$

$X, Y, Z$ coordinate directions defined in Fig. 1 .

\section{Greek Symbols}

$\alpha \quad$ thermal diffusion factor

$\beta \quad$ temperature coefficient of density $(\partial \rho / \partial T)$

$\rho$ density of the mixture

$\Delta \quad$ degree of concentration, $\Delta=\left|C_{T}-C_{B}\right|$

$\lambda$ separation potential defined by Eq. (13)

$\mu \quad$ viscosity of the mixture

\section{Subscripts}

$\begin{array}{ll}T & \text { top } \\ B & \text { bottom } \\ \infty & \text { steady-state }\end{array}$

\section{Acknowledgments}

The authors would like to express their gratitude to Eng Renato Morgado (Universidade do Minho, Portugal) for helpful discussions concerning the present paper, and to NATO Scientific Affairs Division and INIC (Instituto Nacional de Investigação Cientifica, Portugal) for the provision of research grants that made this work possible.

\section{REFERENCES}

1. M. F. Morgado, J. de D. Pinheiro, and J. J. Romero, Proc. Int. Chem. Eng. Conf., CHEMPOR'78, Braga, Portugal, September 1978, Paper 34.1. 
2. T. R. Bott and M. Whysall, J. Chem. Eng. Jpn., 7, 167 (1974).

3. H. M. Yeh and T. Y. Chu, Chem. Eng. Sci., 26, 937 (1971).

4. H. M. Yeh and C. C. Lu, Sep. Sci., $11(5), 455$ (1976).

5. J. J. B. Romero and J. de D. Pinheiro, Chem. Eng. Sci., 30, 1459 (1975).

6. J. de D. Pinheiro, PhD Thesis, University of Birmingham, U.K., 1976.

7. W. Furry, R. C. Jones, and L. Onsager, Phys. Rev., 55, 1083 (1939).

8. J. J. B. Romero, Rev. Fis. Quim. Eng. ULM, A, I, 1 (1970).

9. S. T. Majundar, Phys. Rev., 81,844 (1955).

10. D. Hoffman and A. H. Emery, AIChE J. 9, 653 (1963).

Received by editor January 22, 1981 